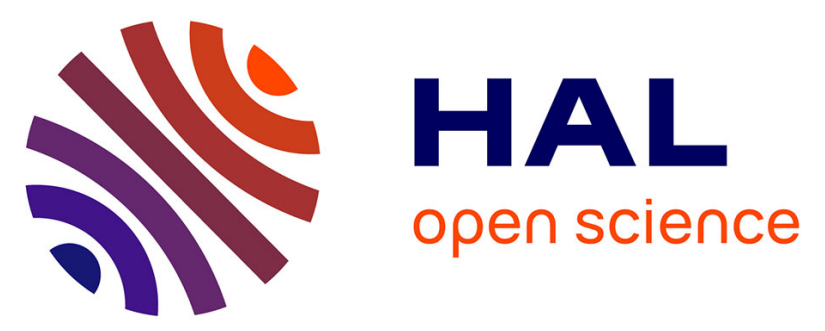

\title{
Insight into magnetic, ferroelectric and elastic properties of strained BiFeO 3 thin films through Mössbauer spectroscopy
}

A Agbelele, D. Sando, I.C. Infante, C Carrétéro, S Jouen, Jean-Marie Le Breton, Alain Barthélémy, B Dkhil, M C Bibes, J Juraszek

\section{To cite this version:}

A Agbelele, D. Sando, I.C. Infante, C Carrétéro, S Jouen, et al.. Insight into magnetic, ferroelectric and elastic properties of strained $\mathrm{BiFeO} 3$ thin films through Mössbauer spectroscopy. Applied Physics Letters, 2016, 109 (109), pp.042902. 10.1063/1.4959790 . hal-01385226

\author{
HAL Id: hal-01385226 \\ https://hal.science/hal-01385226
}

Submitted on 24 Aug 2020

HAL is a multi-disciplinary open access archive for the deposit and dissemination of scientific research documents, whether they are published or not. The documents may come from teaching and research institutions in France or abroad, or from public or private research centers.
L'archive ouverte pluridisciplinaire HAL, est destinée au dépôt et à la diffusion de documents scientifiques de niveau recherche, publiés ou non, émanant des établissements d'enseignement et de recherche français ou étrangers, des laboratoires publics ou privés. 


\section{Insight into magnetic, ferroelectric and elastic properties of strained $\mathrm{BiFeO}_{3}$ thin films through Mössbauer spectroscopy}

Cite as: Appl. Phys. Lett. 109, 042902 (2016); https://doi.org/10.1063/1.4959790

Submitted: 02 May 2016. Accepted: 09 July 2016 . Published Online: 26 July 2016

A. Agbelele, D. Sando, I. C. Infante (D), C. Carrétéro, S. Jouen, J.-M. Le Breton, A. Barthélémy, B. Dkhil, M. Bibes, and J. Juraszek
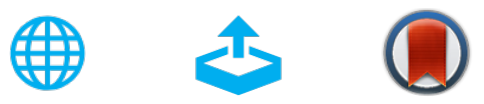

View Online

Export Citation

\section{ARTICLES YOU MAY BE INTERESTED IN}

A multiferroic on the brink: Uncovering the nuances of strain-induced transitions in $\mathrm{BiFeO}_{3}$ Applied Physics Reviews 3, 011106 (2016); https://doi.org/10.1063/1.4944558

Structural, magnetic, and ferroelectric properties of T-like cobalt-doped $\mathrm{BiFeO}_{3}$ thin films APL Materials 6, 026102 (2018); https://doi.org/10.1063/1.5011783

Bulk photovoltaic effect in monodomain $\mathrm{BiFeO}_{3}$ thin films

Applied Physics Letters 110, 183902 (2017); https://doi.org/10.1063/1.4983032

\section{Lock-in Amplifiers up to $600 \mathrm{MHz}$}
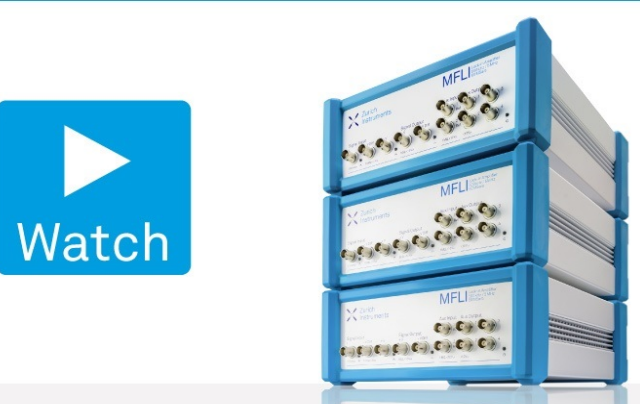


\title{
Insight into magnetic, ferroelectric and elastic properties of strained $\mathrm{BiFeO}_{3}$ thin films through Mössbauer spectroscopy
}

\author{
A. Agbelele, ${ }^{1}$ D. Sando, ${ }^{2,3}$ I. C. Infante,${ }^{4}$ C. Carrétéro, ${ }^{2}$ S. Jouen, ${ }^{1}$ J.-M. Le Breton, ${ }^{1}$ \\ A. Barthélémy, ${ }^{2}$ B. Dkhil, ${ }^{4} \mathrm{M}$. Bibes,${ }^{2}$ and J. Juraszek ${ }^{1, a)}$ \\ ${ }^{1}$ Normandie Univ., UNIROUEN, INSA Rouen, CNRS, Groupe de Physique des Matériaux, 76000 Rouen, \\ France \\ ${ }^{2}$ Unité Mixte de Physique CNRS/Thales, 1 av. Fresnel, 91767 Palaiseau \& Université Paris-Sud, 91405 Orsay, \\ France \\ ${ }^{3}$ School of Materials Science and Engineering, University of New South Wales, 2052 Sydney, Australia \\ ${ }^{4}$ Laboratoire SPMS, Centrale-Supélec, CNRS-UMR 8580, Université Paris-Saclay, 92290 Châtenay-Malabry, \\ France
}

(Received 2 May 2016; accepted 9 July 2016; published online 26 July 2016)

\begin{abstract}
We have studied the magnetic order of highly strained (001)-oriented $\mathrm{BiFeO}_{3}$ (BFO) thin films using ${ }^{57} \mathrm{Fe}$ Conversion Electron Mössbauer Spectrometry. From $90 \mathrm{~K}$ to $620 \mathrm{~K}$ the films exhibit a collinear antiferromagnetic structure, in contrast with the cycloidal structure observed in bulk BFO. Moreover, we find that both the planar magnetic anisotropy for compressive strain and out-of-plane anisotropy for tensile strain persist from $90 \mathrm{~K}$ up to the Néel temperature $\left(T_{\mathrm{N}}\right)$, which itself shows only a weak strain dependence. An analysis of the line asymmetry of the paramagnetic doublet for temperatures above $T_{\mathrm{N}}$ is used to reveal the strain-dependent rotation of the polarization direction, consistent with previous observations. Our results show that the lattice dynamics in BFO films are strongly strain-dependent, offering avenues toward acoustic phonon devices. Finally, we use the versatility of Mössbauer spectroscopy technique to reveal various multi-property features including magnetic states, polarization direction and elastic strain. Published by AIP Publishing.

[http://dx.doi.org/10.1063/1.4959790]
\end{abstract}

\begin{abstract}
Among multiferroics, materials which possess coexisting ferroelectric and magnetic order, $\mathrm{BiFeO}_{3}$ (BFO) is currently the most widely studied due to its rather remarkable multifunctional properties. Indeed, bulk BFO is a robust ferroelectric with a high Curie temperature $T_{\mathrm{C}}=1143 \mathrm{~K}$ (Ref. 1) and is antiferromagnetic below its Neel temperature $T_{\mathrm{N}}=643 \mathrm{~K}^{2}{ }^{2}$ Moreover, these orders are coupled, and control of magnetic properties by an electric field has been demonstrated in single crystals ${ }^{3,4}$ as well as in thin layers. ${ }^{5,6}$ This coupling may lead to the conception of electrically addressable magnetic memories. ${ }^{7}$ Additionally, BFO displays very large polarization $\left(\sim 100 \mu \mathrm{C} / \mathrm{cm}^{2}\right)^{8-11}$ as well as low band gap $(2.6 \mathrm{eV})$ opening the way for many other applications beyond spintronics. ${ }^{12}$

The magnetic structure of bulk BFO is G-type antiferromagnetic superimposed with a long-range cycloidal spin modulation. ${ }^{3,13}$ Early studies demonstrated that the cycloid can be destroyed in thin films due to a magnetoelastic effect induced by strain. ${ }^{14,15}$ However, later neutron diffraction studies on partially relaxed films showed the presence of the cycloid. ${ }^{16}$ To gain deeper insight into the influence of strain on the spin structure, Sando et al. ${ }^{17}$ established the magnetic phase diagram of BFO films deposited on various substrates imposing a wide range $(\sim 3.6 \%)$ of strains. They demonstrated that the cycloid persists at low strain, while collinear magnetic order is stable at high compressive and tensile strains. In addition, the work showed that a rotation of the
\end{abstract}

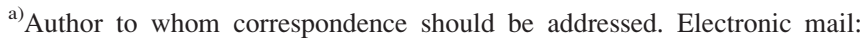
jean.juraszek@univ-rouen.fr
}

spin direction from in-plane to out-of-plane occurs when strain goes from compressive to tensile.

Temperature-dependent studies on single-crystal BFO have evidenced several anomalies below room temperature, via Raman spectroscopy ${ }^{18,19}$ and dielectric susceptibility measurements. ${ }^{20}$ It has been suggested that these anomalies are associated to a spin reorientation transition. However, neutron diffraction studies show no evidence of a change in magnetic structure in this temperature range. ${ }^{21-23}$ Surprisingly, temperature-dependent studies of magnetic order in BFO thin layers up to the Néel temperature $\left(T_{\mathrm{N}}\right)$ are sparse, and the few works that do consider this transition have for the most part utilized indirect techniques. Indeed, while the strong compressive epitaxial strain-induced socalled T-like phase of BFO films has been shown to have a $T_{\mathrm{N}}$ close to room temperature, ${ }^{24-27}$ the magnetic phase transition of the less-strained R-like phase remains unclear due to the use of such indirect techniques. Furthermore, the reported value of $640 \mathrm{~K}$, close to the bulk value, is typically not reached during measurements. ${ }^{24,28,29,48}$

Finally, it is worth mentioning that it was recently shown that $\mathrm{BFO}$ is also an attractive candidate for ultrahigh frequency acoustic devices ${ }^{30}$ by virtue of the possibility to photo-generate ultrafast coherent acoustic phonons. ${ }^{31,32}$ Unfortunately, this property has thus far been poorly explored, and very few studies on BFO have considered lattice dynamics, ${ }^{33,34}$ despite their key role in sound propagation.

In this Letter, we investigate the magnetic phase transition and lattice dynamics of epitaxial $\mathrm{BiFeO}_{3}$ thin films using ${ }^{57} \mathrm{Fe}$ Conversion Electron Mössbauer Spectrometry (CEMS). ${ }^{35}$ Through sensitive direct measurements over the 


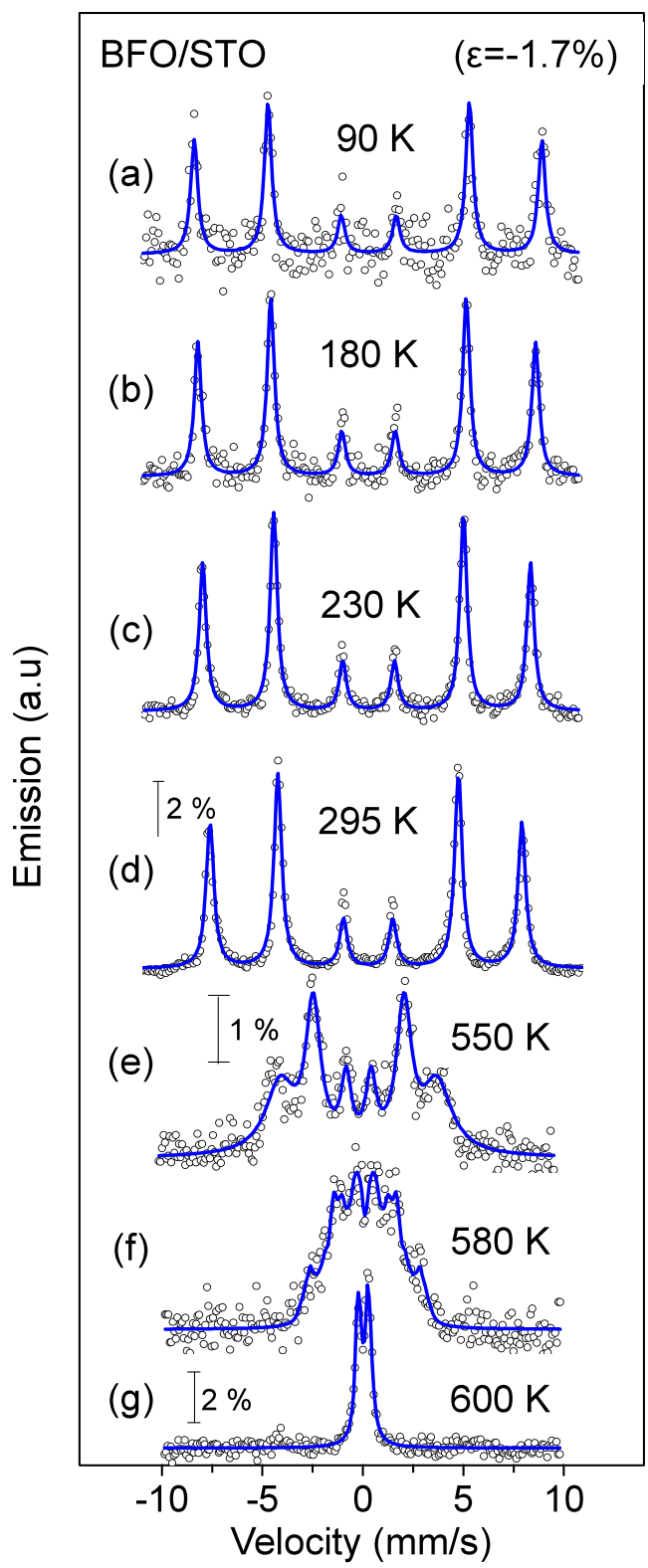

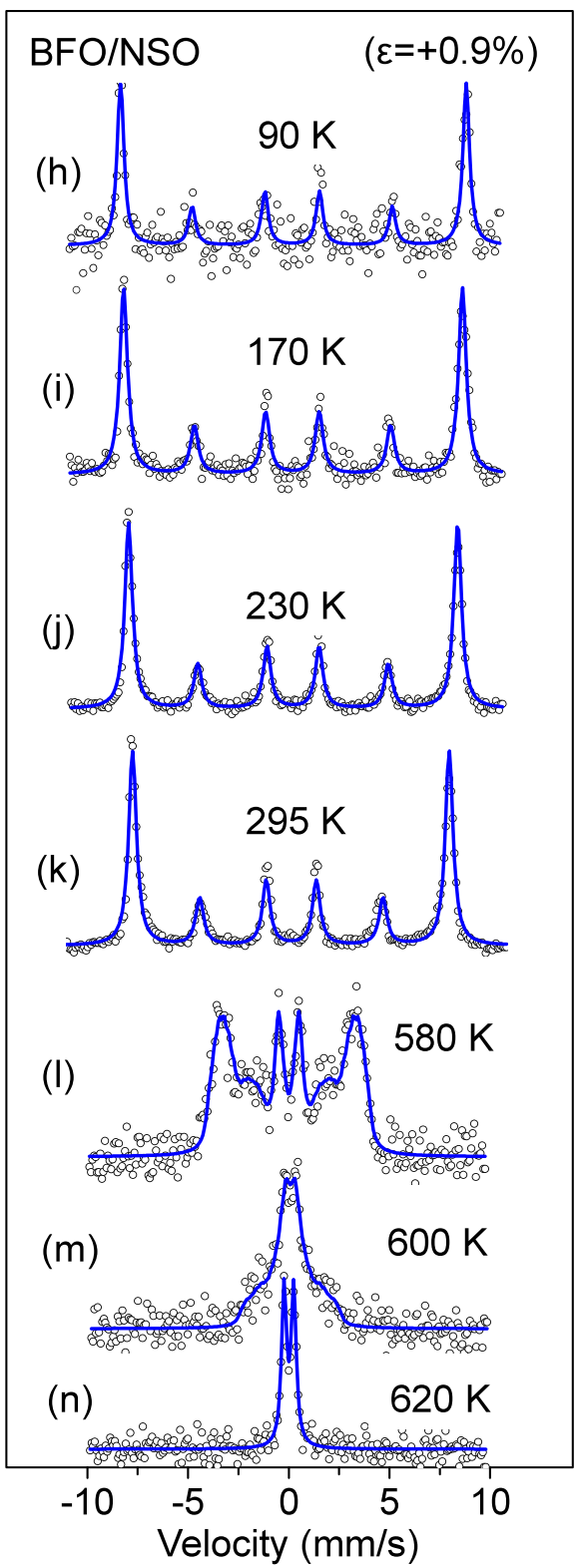

FIG. 1. Mössbauer spectra at various temperatures for epitaxial BFO films grown on STO (a)-(g) and NSO (h)-(n). The data are shown as symbols and lines are best fits to the data. temperature range 90-620 K, i.e., temperatures both below and above the antiferromagnetic-paramagnetic transition, we find that the Néel temperature hardly varies with strain. Furthermore, our analysis of the paramagnetic doublet which appears above $T_{\mathrm{N}}$ is used to reveal the well-known straindependent rotation of the ferroelectric polarization. Finally, interpretation of the data in the context of a Debye model shows that compressive strain increases the sound velocity in BFO films by a factor of 2 relative to the bulk.

The samples used for this study were $70 \mathrm{~nm}$ thick BFO (001) epitaxial layers on $\mathrm{SrTiO}_{3}$ (STO) and $\mathrm{NdScO}_{3}$ (NSO) single crystal substrates, grown by pulsed laser deposition (PLD) (Ref. 36). To enhance the signal in Mössbauer experiments, the films were grown using a $\sim 100 \%{ }^{57} \mathrm{Fe}$ enriched target. The structural characteristics of these samples are comparable to those reported in Ref. 28. Importantly, the films are fully strained on their respective substrates, with the epitaxial strain being $-1.7 \%$ (compressive) for STO and $+0.9 \%$ (tensile) for NSO.

Mössbauer spectroscopy was performed using a $\mathrm{He}-\mathrm{CH}_{4}$ gas-flow proportional counter mounted inside a closed cycle
Janis cryostat for low temperature measurements. ${ }^{37}$ High temperature measurements were performed by heating the sample by a resistive wire placed behind the holder. $\gamma$ photons emitted by $50 \mathrm{mCi}{ }^{57} \mathrm{Co}$ radioactive source in a $\mathrm{Rh}$ matrix in constant acceleration mode were used. The obtained spectra provide information on the electronic density at the nuclei (through the so-called isomer shift), an electric field gradient (EFG) (quadrupole splitting) and the magnetic environment (magnetic hyperfine field). ${ }^{35}$

Figure 1 displays spectra collected under normal incidence at various temperatures for BFO/STO [Figs. 1(a)-1(g)] and BFO/NSO [Figs. 1(h)-1(n)]. At $T=90 \mathrm{~K}$, the spectra for both samples exhibit a symmetric six-line magnetic hyperfine pattern with a hyperfine field $B_{\mathrm{hf}} \sim 54 \mathrm{~T}$ indicating collinear magnetic order for BFO at this temperature. The line intensity ratio $R_{23}$ of the second and third lines is close to 4.0 and 0.8 for $\mathrm{BFO} / \mathrm{STO}$ and $\mathrm{BFO} / \mathrm{NSO}$, respectively, evidencing an in-plane and out-of-plane orientation of the antiferromagnetic vector, respectively. Upon an increase of temperature to $300 \mathrm{~K}$, a decrease in the overall splitting of the outer emission lines (which measures the average 


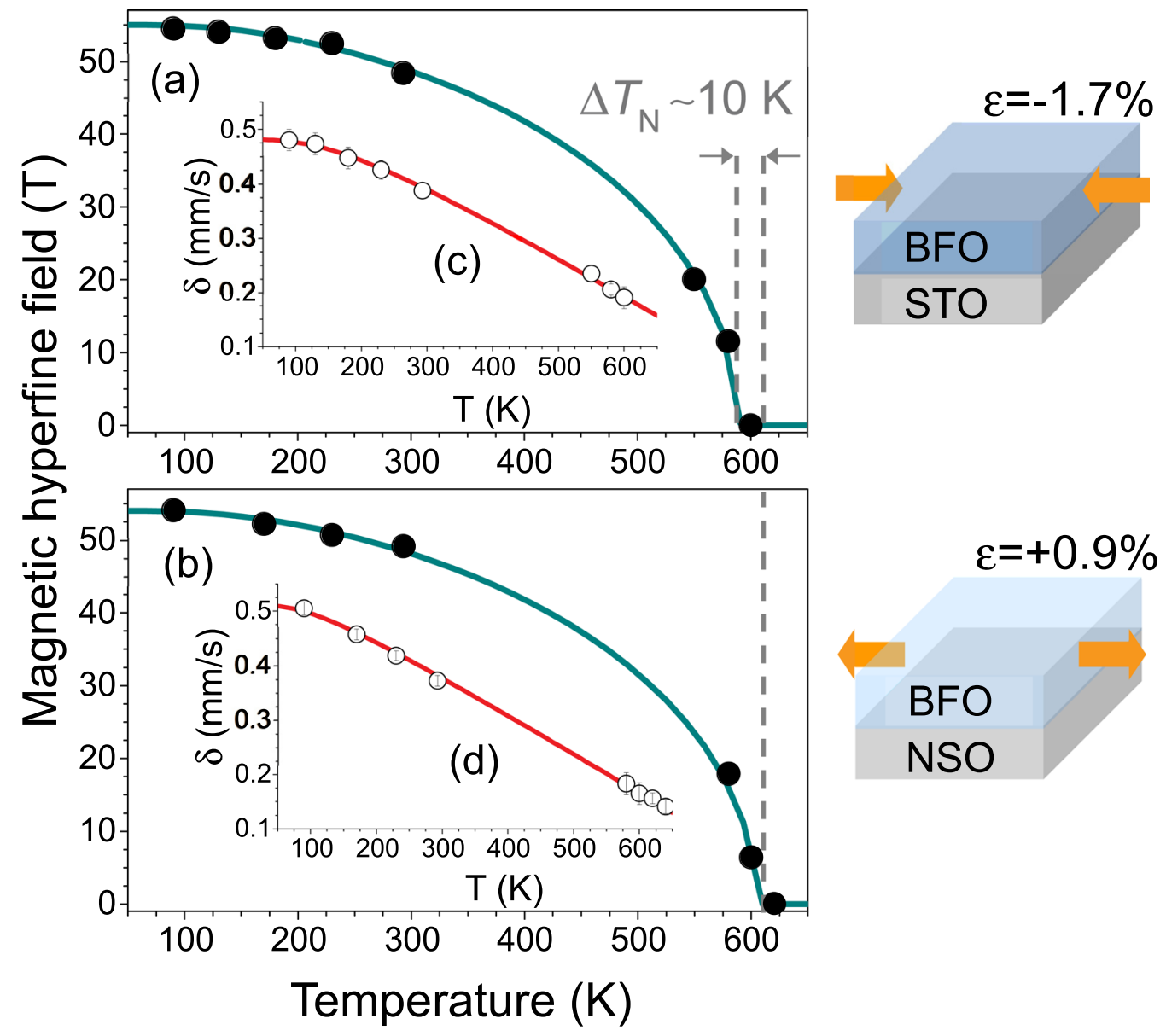

FIG. 2. Temperature dependence of the hyperfine field for BFO/STO (a) and BFO/NSO (b). The green solid lines are Brillouin functions for Fe ${ }^{3+}(S=5 / 2)$ fitted to the data (dots). Isomer shift $(\delta$ ) versus temperature (T) for BFO/STO and BFO/NSO are shown in the insets. The red lines denote the best fits to the data (dots) using a Debye model.

effective $B_{\mathrm{hf}}$ at the nucleus) is observed, reflecting a progressive disorder of the magnetic moments resulting from the reduced strength of the exchange interactions. For both samples, the magnetic hyperfine field and isomer shift values at room temperature are close to $48 \mathrm{~T}$ and $0.37 \mathrm{~mm} \mathrm{~s}^{-1}$, respectively, characteristic of $\mathrm{Fe}^{3+}$ ions in octahedral coordination for $R$-like BFO. ${ }^{17,24,38}$ At $T=550 \mathrm{~K}$, the spectrum for $\mathrm{BFO} /$ STO displays significant line broadening, the result of a distribution of the hyperfine field, and signaling the approach of a magnetic phase transition. The same behavior is observed for $\mathrm{BFO} / \mathrm{NSO}$ at $580 \mathrm{~K}$. Upon further increases in temperature, the spacing between the outer emission lines continues to diminish.

Between 580 and $600 \mathrm{~K}$, the magnetic component disappears completely from the spectrum of BFO/STO, indicating a transition to the paramagnetic phase. For BFO/NSO, on the other hand, this transition appears between 600 and $620 \mathrm{~K}$. For both samples, in the magnetic phase, the ratio $R_{23}$ shows no modification with temperature, suggesting that no spin reorientation occurs over the temperature range of our measurements. These results are in contrast with Raman spectroscopy $^{18,19}$ and dielectric susceptibility measurements ${ }^{20}$ on bulk BFO in which anomalies were observed at $\sim 140 \mathrm{~K}$, and which were attributed to spin reorientation.

Figure 2 presents the temperature dependence of the average $B_{\mathrm{hf}}$ for both samples along with fits using a mean field model based on the Brillouin function. For BFO/STO
[Fig. 2(a)], we find $T_{\mathrm{N}}=590 \mathrm{~K}$ while $T_{\mathrm{N}}=605 \mathrm{~K}$ is obtained for BFO/NSO. These values are slightly lower than the accepted value for bulk $\mathrm{BFO}^{2}(643 \mathrm{~K})$, probably due to the change of symmetry and/or anisotropy in films, ${ }^{13}$ and show a rather weak dependence on epitaxial strain.

In the paramagnetic phase above the Néel temperature (Fig. 1) where only quadrupolar hyperfine interactions remain, a quadrupole-split doublet is observed in the CEMS spectra. This doublet indicates the presence of an electric field gradient in the films, consistent with the ferroelectric character of BFO persisting above $T_{\mathrm{N}}$. Another important feature of the CEMS spectra is the clear asymmetry of the paramagnetic doublet above $T_{\mathrm{N}}$. The relative intensity of these doublets is different for the two samples, implying a dependence on strain. In the general case, three factors may influence the relative line intensities of a paramagnetic doublet: ${ }^{39-41}$

(i) A distribution of quadrupole splitting $\Delta E_{Q}$ due to the presence of non-equivalent Fe-sites;

(ii) lattice vibrational anisotropy (the GoldanskiiKaryagin effect); and/or

(iii) a preferred orientation of the electric field gradient (EFG) (texture).

Taking into account $x$-ray diffraction data ${ }^{28}$ and the fact that the Goldanskii-Karyagin effect is very rare and has never been observed in this system, we consider the first two 
origins unlikely. We therefore argue that the asymmetry in the spectra is attributed to a texture effect. Assuming axial symmetry, the angular dependence of the relative line intensity $\left(R_{q}\right)$ in the quadrupole interaction is given by ${ }^{41}$

$$
R_{q}=\frac{1+\cos ^{2} \theta_{q}}{5 / 3-\cos ^{2} \theta_{q}},
$$

where $\theta_{q}$ is the angle between the direction of the $\gamma$-ray and the principal axis of the EFG. We find $\theta_{q}=47^{\circ}$ for compressive strain (BFO/STO) and $\theta_{q}=59^{\circ}$ for tensile strain (BFO/ NSO). These values enclose $54.74^{\circ}$ corresponding to the angle between the [001] and the direction of the polarization in bulk BFO which is along [111]. Furthermore, our extracted EFG directions are in reasonable agreement with first-principles predictions of the polarization direction for these strain levels: ${ }^{42} 41.6^{\circ}$ for $-1.7 \%$ strain and $57.4^{\circ}$ for $+0.9 \%$ strain. We point out here that for both samples we obtain $\Delta E_{Q} \sim 0.44 \mathrm{~mm} \mathrm{~s}^{-1}$, close to the value obtained for bulk BFO, ${ }^{33,43}$ and consistent with a constant total polarization. On the whole, the results here are consistent with the previous observations that when strain goes from tensile to compressive, a progressive rotation of the polarization vector towards the out-of-plane direction occurs, while there is a negligible change in the vector magnitude. We have thus shown that remarkably, information on the polarization direction in BFO thin films can be deduced from Mössbauer spectra analysis, expanding the investigation possibilities of such technique.

To explore the effect of strain on lattice dynamics in $\mathrm{BFO}$, we consider the temperature dependence of the isomer shift of BFO/STO $(\varepsilon=-1.7 \%)$ and BFO/NSO $(\varepsilon=-0.9 \%)$. The isomer shift $(\delta)$ typically consists of two terms

$$
\delta=\delta_{0}+\delta_{\text {sod }},
$$

where $\delta_{0}$ is the chemical shift, which is proportional to the $s$ electron density at the Fe nucleus, and $\delta_{\text {sod }}$ is the secondorder Doppler shift, which is related to the atomic mean square displacement; and thus strongly temperature dependent. According to the Debye model, the temperature dependence of $\delta_{\text {sod }}$ is given by ${ }^{44-46}$

$$
\delta_{s o d}(T)=\frac{-3 k T}{2 m c}\left[\frac{3 \theta_{D}}{8 T}+3\left(\frac{T}{\theta_{D}}\right)^{3} \int_{0}^{\frac{\theta_{D}}{T}} \frac{x^{3}}{e^{x}-1} d x\right],
$$

where $m$ is the mass of the ${ }^{57} \mathrm{Fe}$ nucleus, $k$ is the Boltzmann constant, $c$ is the speed of light, $x=\hbar \omega / k T$, and $\theta_{D}$ is the Debye temperature.

The evolution of the isomer shift over temperature for the two samples, along with their fits using the Debye model, are plotted in the insets of Fig. 2. In both cases, we observe a gradual decrease of $\delta$ upon increasing T over the whole temperature range, indicating a single phase of BFO. From the fits, we determine the Debye temperature $\theta_{\mathrm{D}}=(626 \pm 21) \quad \mathrm{K}$ and $\theta_{\mathrm{D}}=(375 \pm 22) \mathrm{K}$ for $\mathrm{BFO} / \mathrm{STO}$ and $\mathrm{BFO} / \mathrm{NSO}$, respectively. This difference reflects a prominent strain effect on the lattice dynamic in BFO. The value obtained for tensile strain is close to the bulk value $e^{33}(340 \pm 50) \mathrm{K}$ while, on the other hand, compressive strain induces a rather high value of $\theta_{\mathrm{D}}$. The sound velocity $\left(v_{s}\right)$ in a crystal is linked to its Debye temperature by

$$
\theta_{\mathrm{D}}=\frac{h v_{s}}{k}\left(\frac{3 N}{4 \pi V}\right)^{1 / 3}
$$

where $N / V$ is atom concentration.

Using Equation (3), we deduce the sound velocity in our films: $v_{s, \mathrm{NSO}}=4.96 \mathrm{~km} / \mathrm{s}$ and $v_{s, \mathrm{STO}}=8.25 \mathrm{~km} / \mathrm{s}$ for $\mathrm{BFO} /$ $\mathrm{NSO}$ and $\mathrm{BFO} / \mathrm{STO}$, respectively. The value obtained for $\mathrm{BFO} / \mathrm{NSO}$ (tensile strain) is close to the reported value for BFO (110), ${ }^{32}$ while compressive strain increases the sound speed by a factor of 2 . Since the sound velocity is dependent on elastic coefficients, ${ }^{30,31}$ we argue that this difference is the result of strong strain-induced modifications of the elastic coefficients in BFO. Indeed, a strong dependence on epitaxial strain of the magnetoelastic coupling coefficients is required to explain the strongly reduced critical field required for cycloid destruction when magnetic fields are applied to BFO films. ${ }^{47}$

In summary, we have shown that the Néel temperature in epitaxial BFO films is lower than in the bulk and that it exhibits a weak dependence on strain. Moreover, we found no evidence for magnetic anomalies below room temperature, in contrast with studies on bulk BFO specimens. We showed that polarization vector direction in BFO depends on epitaxial strain, in good agreement with previous results. Finally, we reported that the lattice dynamics are strongly modified by compressive strain, through which a large increase in sound velocity in BFO up to $8.25 \mathrm{~km} / \mathrm{s}$ is observed. We attribute this modification to epitaxial strains which modify elastic constants in BFO; however, this is not only a possible mechanism to explain this increased velocity, and further investigation is required to establish the underlying physics. Our results highlight that multiferroic BFO, particularly in the form of thin films, is a strong candidate for the development of multifunctional devices combining the unique magnetic, ferroelectric, and elastic properties in this material, and that Mössbauer measurement technique is a versatile tool to provide useful information on the aforementioned properties.

A. Agbelele acknowledges the MESR (Ministère de l'Enseignement Supérieur et de la Recherche, France) for financial support.

${ }^{1}$ J. M. Moreau, C. Michel, R. Gerson, and W. J. James, J. Phys. Chem. Solids 32, 1315 (1971).

${ }^{2}$ S. V. Kiselev, R. P. Ozerov, and G. S. Zhdanov, Sov. Phys. Dokl. 7, 742 (1963).

${ }^{3}$ D. Lebeugle, D. Colson, A. Forget, M. Viret, A. M. Bataille, and A. Gukasov, Phys. Rev. Lett. 100, 227602 (2008).

${ }^{4}$ S. Lee, W. Ratcliff, S. Cheong, and V. Kiryukhin, Appl. Phys. Lett. 92, 192906 (2008).

${ }^{5}$ T. Zhao, A. Scholl, F. Zavaliche, K. Lee, M. Barry, A. Doran, M. P. Cruz, Y. H. Chu, C. Ederer, N. A. Spaldin, R. R. Das, D. M. Kim, S. H. Baek, C. B. Eom, and R. Ramesh, Nat. Mater. 5, 823 (2006).

${ }^{6}$ W. Ratcliff II, Z. Yamani, V. Anbusathaiah, T. R. Gao, P. A. Kienzle, H. Cao, and I. Takeuchi, Phys. Rev. B 87, 140405(R) (2013).

${ }^{7}$ M. Bibes and A. Barthélémy, Nat. Mater. 7, 425 (2008).

${ }^{8}$ D. Lebeugle, D. Colson, A. Forget, and M. Viret, Appl. Phys. Lett. 91, 022907 (2007).

${ }^{9}$ V. V. Shvartsman, W. Kleemann, R. Haumont, and J. Kreisel, Appl. Phys. Lett. 90, 172115 (2007). 
${ }^{10}$ H. Béa, M. Bibes, X.-H. Zhu, S. Fusil, K. Bouzehouane, S. Petit, J. Kreisel, and A. Barthelémy, Appl. Phys. Lett. 93, 072901 (2008).

${ }^{11}$ J. Wang, J. Neaton, H. Zheng, V. Nagarajan, S. Ogale, B. Liu, D. Viehland, V. Vaithyanathan, D. Schlom, U. Waghmare, N. Spaldin, K. Rabe, M. Wuttig, and R. Ramesh, Science 299, 1719 (2003).

${ }^{12}$ D. Sando, A. Barthélémy, and M. Bibes, J. Phys.: Condens. Matter 26, 473201 (2014).

${ }^{13}$ I. Sosnowska, T. Peterlin-Neumaier, and E. Steichele, J. Phys. C: Solid State Phys. 15, 4835 (1982).

${ }^{14}$ I. Sosnowska and A. Zvezdin, J. Magn. Magn. Mater. 140-144, 167 (1995).

${ }^{15}$ F. Bai, J. Wang, M. Wuttig, J. Li, N. Wang, A. P. Pyatakov, A. K. Zvezdin, L. E. Cross, and D. Viehland, Appl. Phys. Lett. 86, 032511 (2005).

${ }^{16}$ H. Béa, M. Bibes, S. Petit, J. Kreisel, and A. Barthélémy, Philos. Mag. Lett. 87, 165 (2007).

${ }^{17}$ X. Ke, P. P. Zhang, S. H. Baek, J. Zarestky, W. Tian, and C. B. Eom, Phys. Rev. B 82, 134448 (2010).

${ }^{18}$ D. Sando, A. Agbelele, D. Rahmedov, J. Liu, P. Rovillain, C. Toulouse, I. C. Infante, A. P. Pyatakov, S. Fusil, E. Jacquet, C. Carrétéro, C. Deranlot, S. Lisenkov, D. Wang, J.-M. Le Breton, M. Cazayous, A. Sacuto, J. Juraszek, A. K. Zvezdin, L. Bellaiche, B. Dkhil, A. Barthélémy, and M. Bibes, Nat. Mater. 12, 641 (2013).

${ }^{19}$ M. Cazayous, Y. Gallais, A. Sacuto, R. de Sousa, D. Lebeugle, and D. Colson, Phys. Rev. Lett. 101, 037601 (2008).

${ }^{20}$ M. K. Singh, R. S. Katiyar, and J. F. Scott, J. Phys.: Condens. Matter 20, 252203 (2008).

${ }^{21}$ S. A. T. Redfern, C. Wang, J. W. Hong, G. Catalan, and J. F. Scott, J. Phys.: Condens. Matter 20, 452205 (2008).

${ }^{22}$ J. Herrero-Albillos, G. Catalan, J. A. Rodriguez-Velamazan, M. Viret, D. Colson, and J. F. Scott, J. Phys.: Condens. Matter 22, 256001 (2010).

${ }^{23}$ M. Ramazanoglu, W. Ratcliff II, Y. J. Choi, S. Lee, S.-W. Cheong, and V. Kiryukhin, Phys. Rev. B 83, 174434 (2011).

${ }^{24}$ I. Sosnowska and R. Przeniosło, Phys. Rev. B 84, 144404 (2011).

${ }^{25}$ I. C. Infante, J. Juraszek, S. Fusil, B. Dupé, P. Gemeiner, O. Diéguez, F. Pailloux, S. Jouen, E. Jacquet, G. Geneste, J. Pacaud, J. Íñiguez, L. Bellaiche, A. Barthélémy, B. Dkhil, and M. Bibes, Phys. Rev. Lett. 107, 237601 (2011).

${ }^{26}$ J. Kreisel, P. Jadhav, O. Chaix-Pluchery, M. Varela, N. Dix, F. Sánchez, and J. Fontcuberta, J. Phys.: Condens. Matter 23, 342202 (2011).

${ }^{27}$ G. J. MacDougall, H. M. Christen, W. Siemons, M. D. Biegalski, J. L. Zarestky, S. Liang, E. Dagotto, and S. E. Nagler, Phys. Rev. B 85, 100406(R) (2012).

${ }^{28}$ W. Siemons, G. J. MacDougall, A. A. Aczel, J. L. Zarestky, M. D. Biegalski, S. Liang, E. Dagotto, S. E. Nagler, and H. M. Christen, Appl. Phys. Lett. 101, 212901 (2012).

${ }^{29}$ I. C. Infante, S. Lisenkov, B. Dupé, M. Bibes, S. Fusil, E. Jacquet, G. Geneste, S. Petit, A. Courtial, J. Juraszek, L. Bellaiche, A. Barthélémy, and B. Dkhil, Phys. Rev. Lett. 105, 057601 (2010).
${ }^{30}$ K.-T. Ko, M. H. Jung, Q. He, J. H. Lee, C. S. Woo, K. Chu, J. Seidel, B.G. Jeon, Y. S. Oh, K. H. Kim, W.-I. Liang, H.-J. Chen, Y.-H. Chu, Y. H. Jeong, R. Ramesh, J.-H. Park, and C.-H. Yang, Nat. Commun. 2, 567 (2011).

${ }^{31}$ M. Lejman, G. Vaudel, I. C. Infante, P. Gemeiner, V. E. Gusev, B. Dkhil, and P. Ruello, Nat. Commun. 5, 4301 (2014).

${ }^{32}$ P. Ruello, T. Pezeril, S. Avanesyan, G. Vaudel, V. Gusev, I. C. Infante, and B. Dkhil, Appl. Phys. Lett. 100, 212906 (2012).

${ }^{33}$ L. Y. Chen, J. C. Yang, C. W. Luo, C. W. Laing, K. H. Wu, J.-Y. Lin, T. M. Uen, J. Y. Juang, Y. H. Chu, and T. Kobayashi, Appl. Phys. Lett. 101, 041902 (2012).

${ }^{34}$ C. Blaauw and F. Van der Woude, J. Phys. C: Solid State Phys. 6, 1422 (1973).

${ }^{35}$ V. S. Pokatilov, A. S. Sigov, and A. O. Konovalova, JETP Lett. 94, 698 (2012).

${ }^{36}$ J. Juraszek, O. Zivotsky, H. Chiron, C. Vaudolon, and J. Teillet, Rev. Sci. Instrum. 80, 043905 (2009).

${ }^{37}$ H. Béa, M. Bibes, A. Barthelémy, K. Bouzehouane, E. Jacquet, A. Khodan, J.-P. Contour, S. Fusil, F. Wyczisk, A. Forget, D. Lebeugle, D. Colson, and M. Viret, Appl. Phys. Lett. 87, 072508 (2005).

${ }^{38}$ M. T. Sougrati, M. Jean, S. Jouen, C. Vaudolon, and B. Hannoyer, Hyperfine Interact. 211, 83 (2012).

${ }^{39}$ D. Sando, A. Agbelele, C. Daumont, D. Rahmedov, W. Ren, I. C. Infante, S. Lisenkov, S. Prosandeev, S. Fusil, E. Jacquet, C. Carrétéro, S. Petit, M. Cazayous, J. Juraszek, J.-M. Le Breton, L. Bellaiche, B. Dkhil, A. Barthélémy, and M. Bibes, Trans. R. Soc. A 372, 20120438 (2014).

${ }^{40}$ V. I. Goldanskii, E. F. Makarov, and V. V. Khrapov, Phys. Lett. 3, 344 (1963).

${ }^{41}$ Y. Calage, H. Duroy, G. Ferey, and F. Varret, Eur. J. Solid State Inorg. Chem. 27, 633 (1990).

${ }^{42}$ H. D. Pfannes and U. Gonser, Appl. Phys. 1, 93 (1973).

${ }^{43}$ C. Daumont, W. Ren, I. C. Infante, S. Lisenkov, J. Allibe, C. Carrétéro, S. Fusil, E. Jacquet, T. Bouvet, F. Bouamrane, S. Prosandeev, G. Geneste, B. Dkhil, L. Bellaiche, A. Barthélémy, and M. Bibes, J. Phys.: Condens. Matter 24, 162202 (2012).

${ }^{44}$ D. Lebeugle, D. Colson, A. Forget, M. Viret, P. Bonville, J. F. Marucco, and S. Fusil, Phys. Rev. B 76, 024116 (2007).

${ }^{45}$ J. M. D. Coey, G. A. Sawatzky, and A. H. Morrish, Phys. Rev. 184, 334 (1969).

${ }^{46}$ J. Cieslak, B. F. O. Costa, S. M. Dubiel, M. Reissner, and W. Steiner, J. Phys.: Condens. Matter 17, 2985 (2005).

${ }^{47}$ H. Keller and I. M. Savic, Phys. Rev. B 28, 2638 (1983).

${ }^{48}$ A. Agbelele, D. Sando, C. Toulouse, C. Paillard, R. D. Johnson, R. Rüffer, A. F. Popkov, C. Carrétéro, P. Rovillain, J.-M. Le Breton, B. Dkhil, M. Cazayous, Y. Gallais, M.-A. Measson, A. Sacuto, P. Manuel, A. K. Zvezdin, A. Barthélémy, J. Juraszek, and M. Bibes, Adv. Mater. (submitted). 